\title{
Primary spinal melanoma: illustrative case
}

\author{
Hanna House, BS, Jacob Archer, MD, and Jamie Bradbury, MD \\ Department of Neurological Surgery, Indiana University School of Medicine, Indianapolis, Indiana
}

BACKGROUND Primary spinal melanoma is extremely rare, accounting for $\sim 1 \%$ of all primary melanomas. Typically presenting insidiously in the thoracic spinal cord, primary spinal melanomas can have an acute presentation due to their propensity to hemorrhage.

OBSERVATIONS Despite its rarity, primary spinal melanoma should be included in the differential diagnosis when a hemorrhagic pattern of T1 and T2 intensities is seen on magnetic resonance imaging. Furthermore, the complete diagnosis is crucial because the prognosis of a primary spinal melanoma is considerably more favorable than that of a primary cutaneous melanoma with metastatic spread.

LESSONS Resection is the treatment of choice, with some authors advocating for postoperative chemotherapy, immunotherapy, and/or radiation. We describe a case of acute quadriplegia from hemorrhagic primary spinal melanoma requiring resection.

https://thejns.org/doi/abs/10.3171/CASE21542

KEYWORDS spinal melanoma; intradural tumor; spinal oncology

Primary malignant melanoma of the spinal cord is a rare finding, accounting for $<1 \%$ of all primary melanomas. ${ }^{1}$ Due to the infrequency of this tumor, there is a paucity of literature regarding its various presentations as well as the best diagnostic and treatment methods and what outcomes can be expected, particularly for an acute presentation. We report a case of acute hemorrhagic primary malignant melanoma of the spinal cord, including its presentation, diagnosis, treatment, and outcome.

\section{Illustrative Case}

The patient, a 37-year-old male, had reportedly been complaining of 1 month of neck pain followed by sudden-onset quadriplegia and difficulty breathing. After being intubated at an outside hospital, the patient was transferred to our tertiary referral center after magnetic resonance imaging (MRI) of the cervical spine with and without contrast enhancement demonstrated an intramedullary spinal cord tumor spanning C1-6 with a hemorrhagic component (Fig. 1). Upon arrival, the patient had Medical Research Council (MRC) grade 2/5 strength in the upper and lower extremities on the left side with decreased sensation to light touch. Strength and sensation on the right had improved to full strength. The patient was admitted to the intensive care unit (ICU) and was started on intravenous dexamethasone with a plan to take him to the operating room (OR) within 24 hours.

Further metastatic work-up of MRI of the thoracic and lumbar spine with and without intravenous contrast agent revealed findings of subarachnoid tumor spread along the surface of the spinal cord around T10-11 and conus medullaris (Fig. 2). Computed tomography (CT) of the chest, abdomen, and pelvis did not reveal any other obvious metastatic lesions.

\section{Operative Procedure}

The patient was taken to the $\mathrm{OR}$, and general anesthesia was induced. Baseline motor and sensory evoked potentials were obtained and demonstrated lower motor evoked responses on the left, consistent with his examination. The patient was positioned prone on a Jackson table with hip and chest pads, and his head was placed in a Mayfield head holder. The posterior cervical region was prepared and draped in a sterile fashion. A midline incision was made extending from the base of the occiput to C7. The posterior cervical fascia was exposed, and dissection was performed in a subperiosteal manner exposing C1-5. After drilling troughs, C2-5 was removed en bloc.

ABBREVIATIONS CNS = central nervous system; CT = computed tomography; ICU = intensive care unit; MRC = Medical Research Council; MRI = magnetic resonance imaging; OR = operating room; PET = positron emission tomography.

INCLUDE WHEN CITING Published November 15, 2021; DOI: 10.3171/CASE21542.

SUBMITTED September 21, 2021. ACCEPTED September 27, 2021.

(C) 2021 The authors, CC BY-NC-ND 4.0 (http://creativecommons.org/licenses/by-nc-nd/4.0/). 


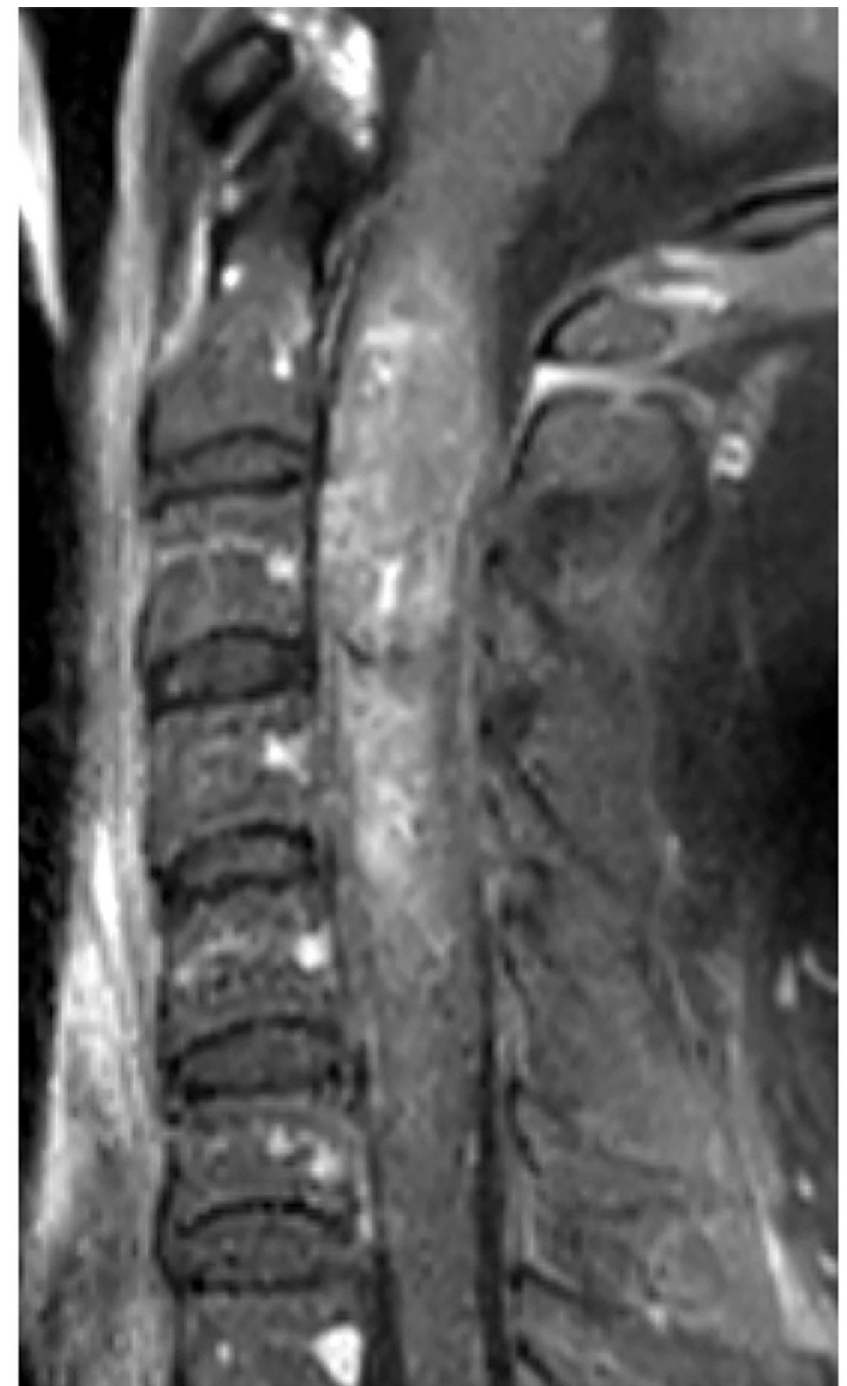

FIG. 1. Sagittal MRI T1-weighted postcontrast image with contrastenhancing intramedullary mass.

The dura was incised in the midline and tacked back with Nurolon sutures. The underlying arachnoid mater was then incised using microscissors. The midline raphe was cauterized using bipolars, and the pia mater was incised using an 11-blade scalpel. Microinstruments were used to dissect in the midline and approached abnormal tissue within a few millimeters.

Microdissection continued circumferentially and both rostrally and caudally to expose the poles with debulking of the tumor using pituitary rongeurs and bipolar electrocautery. Multiple bridging vessels were cauterized and cut with microscissors until finally the tumor was lifted off of the spinal cord. No obvious remaining tumor was visualized around the edges of the resection cavity. There was some reduction in motor and sensory potentials during the resection, but monitorable signals remained at the end of the resection. There was some amount of hematoma that delivered itself during the resection; however, myelotomy was not extended to remove any additional hematoma.

The dura was closed in a watertight manner with a running 4-0 Nurolon suture. The C2-5 laminoplasty was then placed using the

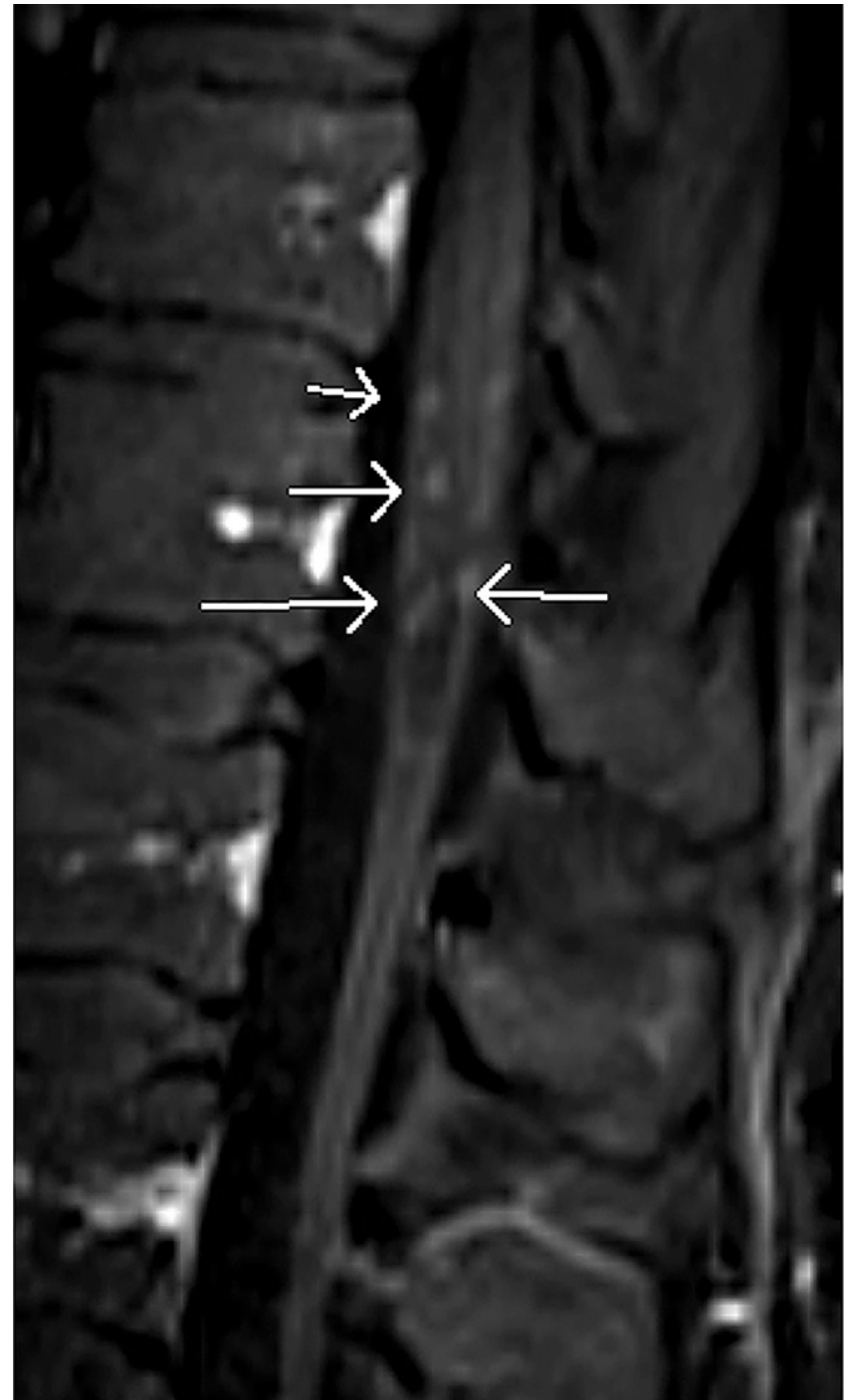

FIG. 2. Sagittal MRI T1-weighted postcontrast image with enhancement along the conus medullaris and cauda equina concerning for leptomeningeal disease. Arrows identify areas of enhancement.

Medtronic centerpiece set. Plates and screws were used to secure the lamina to the lateral mass at each level. Adherus sterile sealant was used around the dural edges, followed by Clorpactin pulse lavage and then bacitracin irrigation. Powdered vancomycin was used inside the wound, and a Hemovac drain was placed. The deep and superficial fascial layers were then closed with interrupted 0 and 2-0 Vicryl sutures, and the skin was stapled closed.

The patient tolerated surgery well and was extubated on postoperative day 2. After surgery, the patient had progressive improvement in sensation and strength to MRC grade $4 / 5$, and postoperative MRI demonstrated gross total resection (Fig. 3). However, the patient developed neurogenic bladder requiring in-and-out catheterization. The patient was transferred out of the ICU after mean arterial pressure goals $>85 \mathrm{~mm}$ $\mathrm{Hg}$ were achieved for 7 days, and he was discharged to an acute rehabilitation center on postoperative day 11 .

Histopathological analysis confirmed melanoma, demonstrating epithelioid and spindle-shaped cells with hyperchromatic nuclei and 


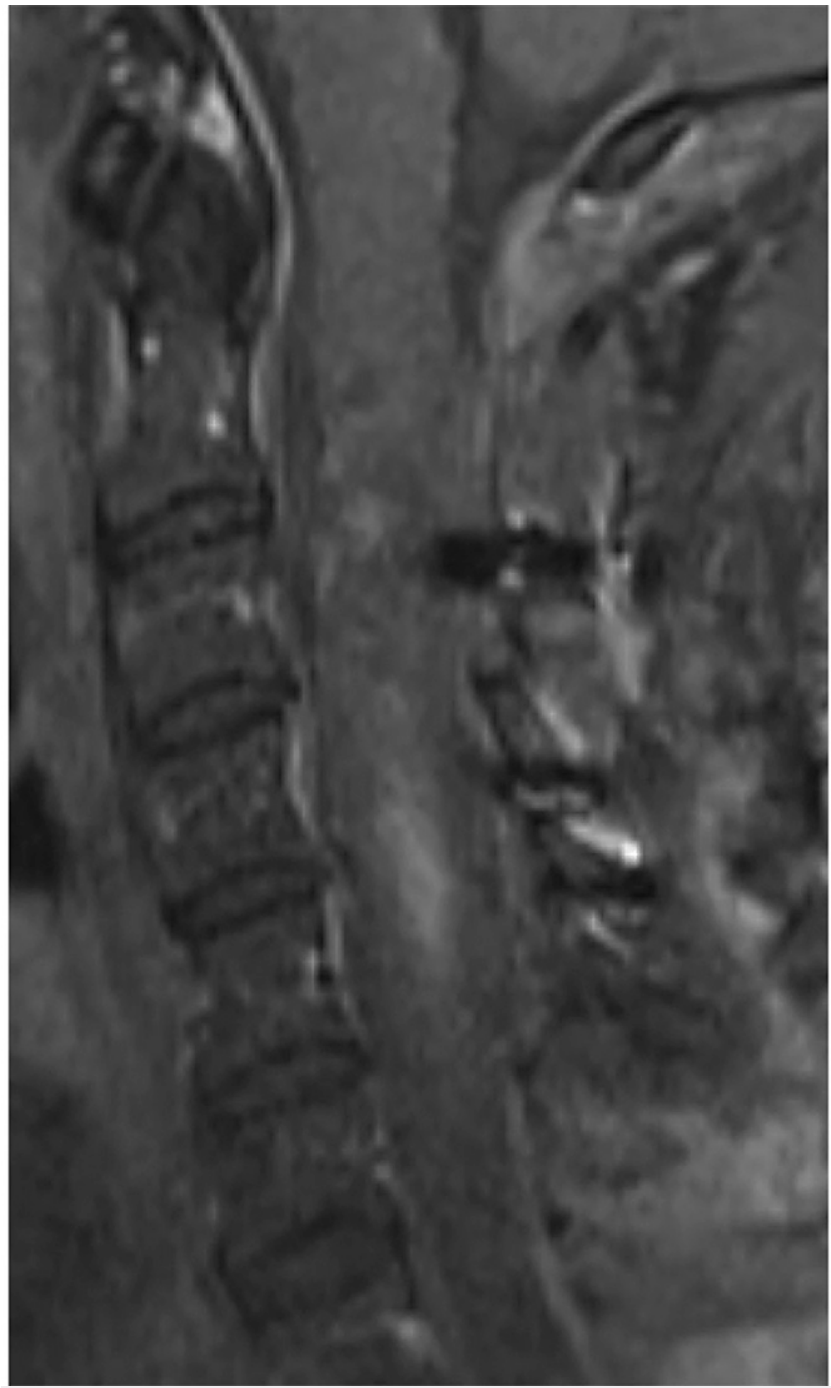

FIG. 3. Sagittal MRI T1-weighted postcontrast image with resolution of intramedullary mass and some residual blood products.

positive staining for CD10, melan-A, and SOX10. The BRAF mutation was not detected.

Follow-up positron emission tomography (PET)-CT was performed and showed no fluorodeoxyglucose-avid metastatic disease. The patient was seen in follow-up by ophthalmology, dermatology, and gastroenterology to find a potential primary source, which was unremarkable. In addition, he was seen in follow-up with radiation oncology and hematology-oncology to receive adjuvant radiation therapy and immunotherapy consisting of nivolumab and ipilimumab, respectively.

At 1-year follow-up, the patient had returned to work full-time. On physical examination, he had some persistent numbness in his legs, but his postoperative weakness had completely resolved. MRI did not show any local recurrence but had some persistent nonspecific leptomeningeal enhancement throughout (Figs. 4 and 5).

\section{Discussion}

The first primary spinal melanoma was described in $1906 .^{2}$ To our knowledge, since then, $<70$ cases of primary spinal cord

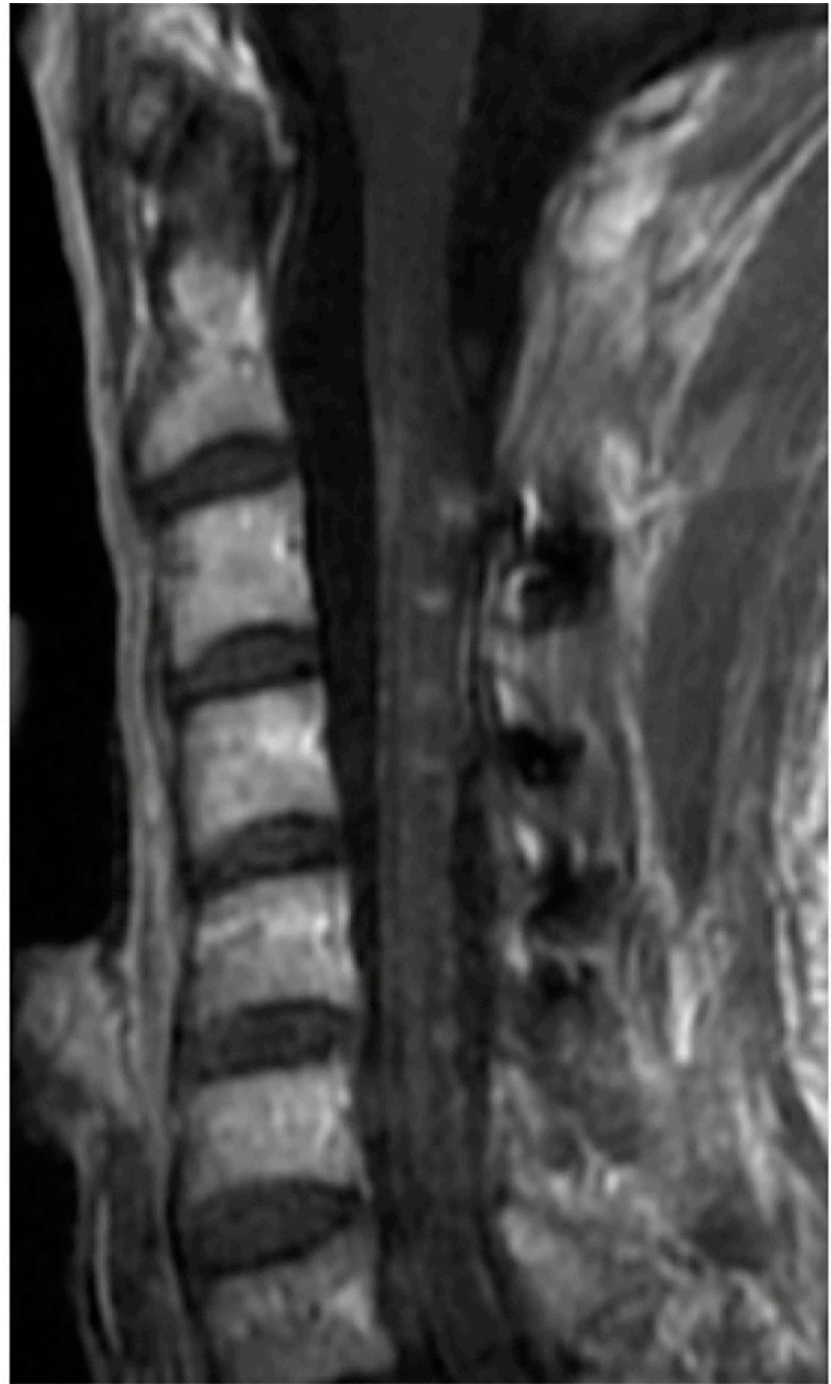

FIG. 4. Sagittal MRI T1-weighted postcontrast image without evidence of any tumor recurrence and resolution of residual hematoma.

melanoma have been reported worldwide. ${ }^{3}$ Most spinal melanomas discovered occur due to metastatic spread from the skin, uvea, or gastrointestinal or vaginal mucosa. ${ }^{3}$ However, melanomas can occur in any anatomical site that contains melanocytes, including the central nervous system (CNS). ${ }^{4}$

\section{Observations}

Primary CNS melanomas account for $\sim 1 \%$ of all melanomas, with the incidence of primary spinal cord melanoma being even rarer. ${ }^{4}$ Among primary spinal cord melanomas, intradural intramedullary tumors are less common, typically comprising $\sim 37.7 \%$ of primary spinal cord melanomas discovered. ${ }^{4}$ Primary spinal cord melanomas most commonly occur in the fifth decade of life and in the middle and lower thoracic regions of the spinal cord $(42 \%),{ }^{3,5}$ with a propensity for involvement of the conus medullaris, ${ }^{6-8}$ followed by cervical $(35 \%)$, thoracolumbar $(12 \%)$, cervicothoracic $(8 \%)$, and lumbar $(4 \%)$ regions. ${ }^{5}$ One case report even described a primary melanoma of the medulla oblongata. ${ }^{9}$ Often, the presenting 


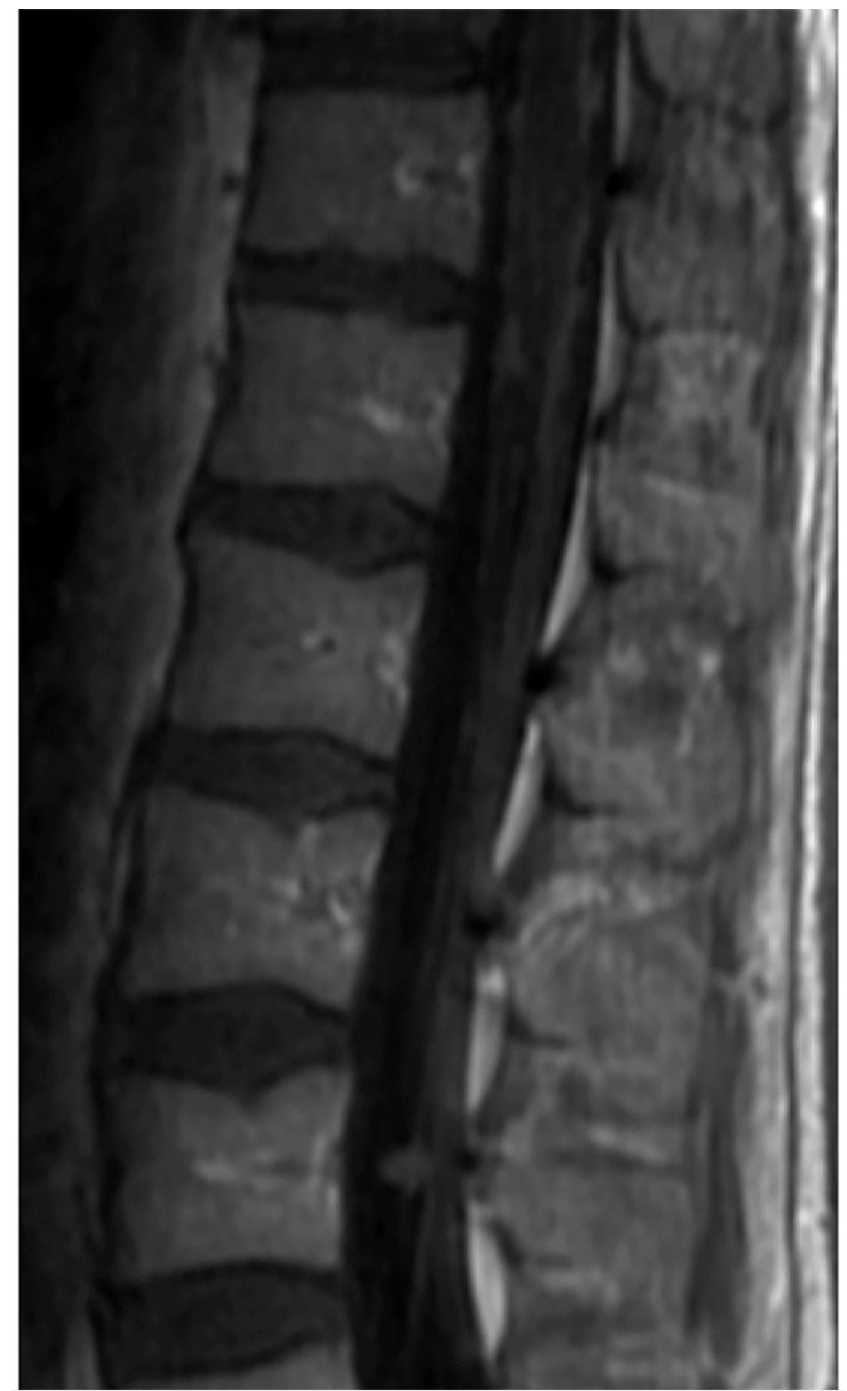

FIG. 5. Sagittal MRI T1-weighted postcontrast image with persistent but stable leptomeningeal enhancement.

symptom is back or neck pain accompanied by a progressive, asymmetrical myelopathy. ${ }^{3}$ The average duration of symptoms before pathological diagnosis was 29 months. ${ }^{10}$ However, acute decompensation can occur due to hemorrhage. ${ }^{11}$ Initial diagnosis requires neuroimaging, with spinal MRI being the best imaging modality due to either melanin's paramagnetic properties or hemorrhagic elements in the tumor. ${ }^{1}$

Typically, these tumors are slightly hyperintense on T1 and iso- to hypointense on T2 with a homogeneous mild enhancement after intravenous gadolinium enhancement. ${ }^{1}$ Further diagnosis, supported by Hayward's criteria, ${ }^{10}$ requires that there be no malignant melanoma outside of the CNS (primary or metastatic) and that the lesion be confirmed pathologically. This consists of ruling out extra-CNS lesions with a fullbody PET-CT scan and thorough dermatologic, ophthalmologic, gastrointestinal, and gynecological evaluations followed by histopathologic confirmation. ${ }^{3}$ This has limitations, however, because achromic cutaneous melanomas exist, and metastatic melanomas of the skin can appear after complete disappearance of a primitive melanoma. ${ }^{2}$ Histologically, melanoma is characterized by large, pleomorphic epithelioid or spindled cells with irregular nuclei, necrosis, and high mitotic index and positive staining for HMB-45 and S100 protein. $^{3}$

\section{Lessons}

Identifying and differentiating primary spinal cord melanoma from metastatic melanoma is important due to its better prognosis. Primary CNS melanomas grow slower and seem to be less malignant than cutaneous melanomas. ${ }^{12}$ Life expectancy for metastatic cutaneous melanomas is typically $<1$ year, whereas the mean survival for primary spinal cord melanomas has been reported at 6 years and 7 months. ${ }^{1,10,13,14}$ Recurrences have been reported, including in the original tumor bed itself and via leptomeningeal dissemination. ${ }^{4}$

There is little research on the management and prognosis of primary spinal cord melanoma. Resection has been advocated as the treatment of choice for primary spinal melanomas. Some authors endorse postoperative chemotherapy or radiotherapy, despite clear evidence of lowering the risk of metastasis. ${ }^{1}$

\section{References}

1. Cetinalp NE, Yildirim AE, Divanlioglu D, Belen D. An uncommon intramedullary tumor: primary spinal cord melanoma. Asian Spine J. 2014;8(4):512-515

2. François $P$, Lioret $E$, Jan $M$. Primary spinal melanoma: case report. Br J Neurosurg. 1998;12(2):179-182.

3. Chatterjee R, Nascimento FA, Heck KA, Ropper AE, Sabichi AL. Primary spinal cord melanoma - an uncommon entity. Can $J$ Neurol Sci. 2019;46(3):348-350.

4. Corrêa DG, Dos Santos RQ, Hygino da Cruz LC Jr. Primary intramedullary malignant melanoma: can imaging lead to the correct diagnosis? J Int Med Res. 2020;48(10):300060520966152.

5. Trinh V, Medina-Flores R, Taylor CL, Yonas H, Chohan MO. Primary melanocytic tumors of the central nervous system: report of two cases and review of literature. Surg Neurol Int. 2014; 5:147.

6. Ebner FH, Roser F, Acioly MA, Schoeber W, Tatagiba M. Intramedullary lesions of the conus medullaris: differential diagnosis and surgical management. Neurosurg Rev. 2009;32(3): 287-301.

7. Farrokh D, Fransen P, Faverly D. MR findings of a primary intramedullary malignant melanoma: case report and literature review. AJNR Am J Neuroradiol. 2001;22(10):1864-1866.

8. Nogueira RM, Cardoso LS, Fonseca L, et al. An uncommon intramedullary tumor: primary medullary cone melanoma. Surg Neurol Int. 2020;11:200.

9. Wu L, Xu Y. Primary spinal intramedullary malignant melanoma involving the medulla oblongata. Spine J. 2016;16(8):e499-e500.

10. Larson TC 3rd, Houser OW, Onofrio BM, Piepgras DG. Primary spinal melanoma. J Neurosurg. 1987;66(1):47-49.

11. Denaro L, Pallini R, Di Muro L, et al. Primary hemorrhagic intramedullary melanoma. Case report with emphasis on the difficult preoperative diagnosis. J Neurosurg Sci. 2007;51(4):181-183.

12. Kolasa M, Jesionek-Kupnicka D, Kordek R, Kolasa P. Primary spinal cord melanoma - a case report. Folia Neuropathol. 2010;48(3) 212-216.

13. Nishihara M, Sasayama T, Kondoh T, Tanaka K, Kohmura E, Kudo $\mathrm{H}$. Long-term survival after surgical resection of primary spinal malignant melanoma. Neurol Med Chir (Tokyo). 2009;49(11): 546-548.

14. Salpietro FM, Alafaci C, Gervasio O, et al. Primary cervical melanoma with brain metastases. Case report and review of the literature. J Neurosurg. 1998;89(4):659-666. 


\section{Disclosures}

The authors report no conflict of interest concerning the materials or methods used in this study or the findings specified in this paper.

\section{Author Contributions}

Conception and design: all authors. Acquisition of data: Archer, House. Analysis and interpretation of data: Archer, House. Drafting the article: House. Critically revising the article: all authors. Reviewed submitted version of manuscript: all authors. Approved the final version of the manuscript on behalf of all authors: Archer. Statistical analysis: House. Administrative/technical/material support: House. Study supervision: House, Bradbury.

\section{Correspondence}

Jacob Archer: Indiana University School of Medicine, Indianapolis, IN. jabarch@iu.edu. 